\title{
Identification and validation of novel EST-SSR markers in olives
}

\author{
Alenka Baruca Arbeiter ${ }^{1}$, Matjaž Hladnik ${ }^{1}$, Jernej Jakše ${ }^{2}$, Dunja Bandelj ${ }^{1}$ *
}

'University of Primorska/Faculty of Mathematics, Natural Sciences and Information Technologies, Glagoljaška ulica, 8 - 6000 - Koper - Slovenia.

¿University of Ljubljana/Biotechnical Faculty - Agronomy Dept., Jamnikarjeva ulica, 101 - 1000 - Ljubljana -

Slovenia.

*Corresponding author <dunja.bandelj@upr.si>

Edited by: Leonardo Oliveira Medici

Received March 16, 2016

Accepted June 05, 2016
ABSTRACT: The olive (Olea europaea L.) is a leading oil crop in the Mediterranean area. Limited information on the inheritance of agronomic significant traits hinders progress in olive breeding programs, which encourages the development of markers linked to the traits. In this study, we report on the development of 46 olive simple sequence repeat (SSR) markers, obtained from 577,025 expressed sequence tags (ESTs) in developing olive fruits generated in the framework of the Slovenian national olive transcriptome project. Sequences were de novo assembled into 98,924 unigenes, which were then used as a source for microsatellites searching. We identified 923 unigenes that contained 984 SSRs among which dinucleotide SSRs (36 \%) were the most abundant, followed by tri- (33\%) and hexa- $(21 \%)$ nucleotides. Microsatellite repeat motif GA (37\%) was the most common among dinucleotides, while microsatellite repeat motif GAA was the most abundant trinucleotide SSR motif (16\%). Gene ontology annotations could be assigned to $27 \%$ of the unigenes. A hundred and ten expressed sequence tag-derived-simple sequence repeats (EST-SSRs) with annotated genes were selected for primer designing and finally, 46 (42\%) polymorphic EST-SSRs were successfully amplified and used to validate genetic diversity among 24 olive varieties. The average number of alleles per locus, observed heterozygosity, expected heterozygosity, and polymorphic information content were 4.5, 0.649, 0.604 and 0.539 , respectively. Twenty-seven EST-SSRs showed good diversity properties and were recommended for further olive genome investigation.

Keywords: expressed sequence tags, microsatellites, genic markers, genetic diversity, functional annotation

\section{Introduction}

Olive (Olea europaea L.) production is the most important agricultural branch in the Mediterranean basin, and olive oil is the main source of fats in the wealthy Mediterranean diet. In addition to high levels of monounsaturated fatty acids, it contains biologically active molecules, including biophenols, squalene, tocopherols, and phytosterols, which have many positive effects on human health (Ali Hashmi et al., 2015; Ghanbari et al., 2012) and are the major contributors to the unique taste of the oil (Cicerale et al., 2009). Consequently, the investigation of genes responsible for the synthesis of these molecules is essential to improve olive oil quality and develop new varieties.

Breeders and the olive industry are currently focused on identifying high-performance genotypes, while the increasing need for diversity of varieties that are well-adopted to changes in the environment, cultivation conditions and consumer requirements dictate the development of new olive varieties (Lavee et al., 2014). For these reasons, the use of new technologies, including new molecular markers, are essential for breeding success and validation of authenticity and traceability of primary products entering the agro-food chain (Corrado, 2016; Pasqualone et al., 2016).

With the improvement and development of next generation sequencing technology, expressed sequence tags (ESTs) are publicly available and represent very useful tool for gene and marker discovery, which are attractive for gene mapping, functional studies, genome annotation and comparative genomics (Ozgenturk et al., 2010; Rudd, 2003). Among them, genic microsatellites, or expressed sequence tag-derived simple sequence repeats (EST-SSRs), have found a special place in plant genetics and breeding of several agricultural plants (Kalia et al., 2011; Varshney et al., 2005). However, a few transcriptome projects on the generation of ESTs in olive have been completed recently (Alagna et al., 2009; Muñoz-Mérida et al., 2013; Ozgenturk et al., 2010; Rešetič et al., 2013), and a limited number of EST-SSRs are currently available in olives (Adawy et al., 2015; De la Rosa et al., 2013; Essalouh et al., 2014). The main purpose of this study was to increase the number of validated EST-SSRs, for the benefit of all interested research groups, via the identification of simple sequence repeats (SSRs) from the transcripts of developing olive fruits of the variety "Istrska belica". The validation and putative functional annotation of a new set of EST-SSRs and their applicability in olive diversity study are reported in this paper.

\section{Materials and Methods}

\section{Identification of EST-SSRs and primer designing}

A total of 577,025 ESTs from developing fruits of the "Istrska belica" olive variety, with an average length of $241 \mathrm{bp}$, were generated using the 454 pyrosequencing methodology (Rešetič et al., 2013) and were assembled by the iAssembler v.1.2.2 (Zheng et al., 2011) into 98,924 unigenes. All cleaned EST sequences (removed taxonomic nonspecific reads and reads shorter than $200 \mathrm{bp}$ ) have been deposited at the SRA database (http://www.ncbi. nlm.nih.gov/sra/SRX215662), and the Transcriptome 
Shotgun Assembly project has been deposited at Databank of Japan/ European Molecular Biology Laboratory (EMBL) Data Library/GenBank under the accession number GDUL00000000 (http://www.ncbi.nlm.nih. gov/nuccore/GDUL00000000). In the current study, all 98,924 assembled sequences were used as a source for microsatellite identification. The mining of microsatellites was performed using the Perl script MIcroSAtelitte (MISA) (http://pgrc.ipk-gatersleben.de/misa). The main criterion for SSR identification was the minimum length, that is, eight repeat units for dinucleotides motif, six repeat units for trinucleotide and tetranucleotide, and four repeat units for pentanuclotide and other higherorder repeats. Sequences containing SSRs longer than 20 nucleotides were first reviewed with the use of Tablet (a next generation sequence assembly viewer) (Milne et al., 2010) in order to exclude all SSR-including sequences that were inappropriate for primer designing according to the following criteria: (a) very short DNA sequence flanking the microsatellite (less than 30 bases) or (b) microsatellite sequence repeat was used by assembler as an overlapping part for the adjacent reads, therefore, there is a probability that this contig is a chimeric one. Sequences were then aligned against National Center for Biotechnology Information (NCBI) non-redundant $(\mathrm{nr})$ protein database using the BLASTX algorithm to determine the putative function $(\mathrm{E}<1 \mathrm{e}-10)$. Only SSR-containing transcripts with annotated genes were identified as candidates for SSR marker development. Primer3 v.4.0.0 tool (Koressaar and Remm, 2007; Untergrasser et al., 2012) with default parameters was used for designation of 110 primer pairs. A single criterion for primer designation was the length of the microsatellite sequence (150 - $200 \mathrm{bp}$ ). A shorter primer in the pair was elongated for the M13 (-21) $18 \mathrm{bp}$ sequence at the $5^{\prime}$ end (5'-TGTAAAACGACGGCCAGT-3') for economic fluorescent labelling (Schuelke, 2000). Integrated DNA Technologies (IDT) synthesized all primers. GenBank Accession numbers, locus names, primer sequences, repeat motifs, SSR locations, annealing temperatures, size ranges and putative functions are listed in Table 1.

\section{Marker validation}

Twenty-four olive varieties ("Arbequina", "Ascolana tenera", "Athena", "Buga", "Cipressino", "Coratina", "Črnica", "Frantoio", "Grignan", "Istrska belica", "Itrana", "Leccino", "Leccio del corno", "Leccione", "Maurino", "Moraiolo", "Nocellara del Belice", "Oblica", "Pendolino", "Picholine", "Samo", "Santa Caterina", "Štorta", "Zelenjak") from a Slovenian collection orchard (N $45^{\circ} 34^{\prime} 29.98^{\prime \prime}$, E $13^{\circ} 46^{\prime} 09.30^{\prime \prime}$ ) were used to evaluate the potential of newly developed EST-SSRs for the olive diversity study. Total DNA was extracted from leaf tissue using the Cetyl trimethylammonium bromide (CTAB) method (Kump and Javornik, 1996) and DNA concentrations were measured with a fluorometer.

All 110 developed primer pairs were used for initial screening in the genotyping procedure of eight olive varieties. The optimal annealing temperature $\left(\mathrm{T}_{\mathrm{a}}\right)$ was experimentally determined for each locus where five different $\mathrm{T}_{\mathrm{a}}$ were tested. The highest annealing temperatures $\left(\mathrm{T}_{\mathrm{a} 1}\right)$ in touchdown polymerase chain reaction (PCR) were set at $60{ }^{\circ} \mathrm{C}, 58^{\circ} \mathrm{C}, 55^{\circ} \mathrm{C}, 53^{\circ} \mathrm{C}$ and $50^{\circ} \mathrm{C}$. Loci with unstable PCR amplification were additionally optimized by raising the DNA concentration, and by increasing the number of cycles in the second step of the amplification. Finally, the amplification of SSRs was performed in a total volume of $15 \mu \mathrm{L}$, containing $1 \times$ supplied PCR buffer, $2 \mathrm{mM} \mathrm{MgCl}, 0.2 \mathrm{mM}$ of each deoxynucleotide (dNTP), $0.2 \mu \mathrm{M}$ of each locus specific primer with one of the primers in pair that was elongated for M13(-21) universal sequence (Schuelke, 2000), $0.25 \mu \mathrm{M}$ of M13(-21) universal primer labelled with 6-FAM, VIC, PET or NED, 0.375 unit of Taq DNA polymerase (Thermo Fischer Scientific, Waltham, USA) and $40 \mathrm{ng}$ of olive DNA. The amplification was performed in a thermal cycler, and the conditions of the two-step PCR were as follows: $94^{\circ} \mathrm{C}$ for $5 \mathrm{~min}$, then 5 cycles at $94{ }^{\circ} \mathrm{C}$ for $45 \mathrm{~s}$, $30 \mathrm{~s}$ at $\mathrm{T}_{\mathrm{a} 1}$ (Table 1), which was lowered by $1{ }^{\circ} \mathrm{C}$ in each cycle, and the extension at $72{ }^{\circ} \mathrm{C}$ for $1 \mathrm{~min} 30 \mathrm{~s}$. The second step of amplification passed through 35 cycles of $30 \mathrm{~s}$ at $94{ }^{\circ} \mathrm{C}, 30 \mathrm{~s}$ at the lowest annealing temperature $\left(\mathrm{T}_{\mathrm{a} 2}\right.$ ) (Table 1), $1 \mathrm{~min} 30 \mathrm{~s}$ at $72{ }^{\circ} \mathrm{C}$, and a final extension at $72{ }^{\circ} \mathrm{C}$ for $10 \mathrm{~min}$. Separation of amplified SSRs was performed in a 3130 Genetic Analyzer, using 500 LIZ size standard. Data were analyzed with Gene Mapper v.4.1 software.

Genetic parameters were calculated for 24 olive varieties over 46 EST-SSR loci that produced clear fragments after amplification. The observed $\left(\mathrm{H}_{\mathrm{o}}\right)$ and expected $\left(\mathrm{H}_{\mathrm{e}}\right)$ heterozygosity, polymorphic information content (PIC), and the frequency of null alleles (F(null)) were calculated using the CERVUS v.3.0.7 computer software. IDENTITY v.1.0 software was used to calculate probability of identity (PI). The effective number of alleles $\left(\mathrm{n}_{\mathrm{e}}\right)$, number of observed and all possible genotypes and deviations from the Hardy-Weinberg equilibrium (HWE) were calculated using the POPGENE v.1.32 software. The algorithm by Levene (1949) was used for calculation expected genotypic frequencies under random mating, and chi-square $\left(\chi^{2}\right)$ tests were performed for HWE at each locus. Variety-specific alleles were calculated by using MICROSAT software. The AMaCAID script (Caroli et al., 2011) was used to elucidate the minimum number of markers required to distinguish all observed genotypes, including the Model3, and the fixed number of combinations was set at 50,000.

Genetic distances between 24 varieties were calculated using Jaccard's coefficient of similarity. A dendrogram was constructed from the matrix of similarity, using the unweighted pair-group method (UPGMA). For the dendrogram, the correlation coefficient between the distance matrix and the cophenetic values matrix was computed to test the goodness of fit of the cluster analysis using the MXCOMP module of the Mantel test (Mantel, 1967). All calculations were performed using the NTSYS v.2.02 software. 
Table 1 - GenBank Accession numbers, locus names, primer sequences, repeat motifs, simple sequence repeat (SSR) locations in untranslated region (UTR) or in coding sequence (CDS), primers the highest $\left(T_{a 1}\right)$ and the lowest $\left(T_{a 2}\right)$ annealing temperatures in touchdown polymerase chain reaction (PCR), size ranges and putative functions for newly developed expressed sequence tag-derived simple sequence repeats (EST-SSRS).

\begin{tabular}{|c|c|c|c|c|c|c|}
\hline $\begin{array}{l}\text { GenBank } \\
\text { Accession No. }\end{array}$ & Primer sequence (5'-3') & $\begin{array}{l}\text { Repeat } \\
\text { motif }\end{array}$ & $\begin{array}{l}\text { SSR } \\
\text { location }\end{array}$ & $\mathrm{T}_{\mathrm{a} 1}, \mathrm{~T}_{\mathrm{a} 2}$ & $\begin{array}{l}\text { Size range } \\
\quad(\mathrm{bp})\end{array}$ & Putative function; accession code \\
\hline GDUL01003735 & $\begin{aligned} \text { OeUP-01 F: TGTCTCATGCCAGATAAACAG } \\
\text { R: *GCAGCAATCCCACAAACTAA }\end{aligned}$ & (AAG)11 & 5' UTR & 55,50 & $200-221$ & $\begin{array}{l}\text { aquaporin-like protein [Petunia } \times \text { hybrida]; } \\
\text { AAL49750.1 }\end{array}$ \\
\hline GDUL01018604 & $\begin{array}{l}\text { OeUP-02 F: *TTTGTCGCTTGCAGATTCCC } \\
\text { R: CTCCCTTACCAGTTGTCGGA }\end{array}$ & (TGC)7 & CDS & 50,45 & $197-255$ & $\begin{array}{l}\text { ribosomal lysine N-methyltransferase } 3 \text { isoform } \\
\text { X2 [Sesamum indicum]; XP_011076932.1 }\end{array}$ \\
\hline GDUL01058010 & $\begin{array}{c}\text { OeUP-03 F: *TCATACGGGCTAGTCGATCG } \\
\text { R: ATCTGCGCTAGTAACACAT }\end{array}$ & (TC)13(AC)8 & 3' UTR & 50,45 & $166-170$ & $\begin{array}{l}\text { pre-mRNA cleavage factor Im } 25 \mathrm{kDa} \text { subunit } \\
\text { 2-like [Sesamum indicum]; XP_011084411.1 }\end{array}$ \\
\hline GDUL01030423 & $\begin{array}{l}\text { OeUP-04 F: *TCCGAAATCCCAGTAGAGTTTT } \\
\text { R: GCAGCAGAAATCACCAATCCT }\end{array}$ & (TCT)7 & 5' UTR & 50,45 & $196-232$ & $\begin{array}{l}\text { uncharacterized protein LOC104247946 [Nico- } \\
\text { tiana sylvestris]; XP_009802414.1 }\end{array}$ \\
\hline GDUL01046188 & $\begin{aligned} \text { OeUP-05 F: GGCACCAAAGCTAAGCTCAA } \\
\text { R: *CCCGGGAAAGTGTTCTGT }\end{aligned}$ & $(\mathrm{CAG}) 8$ & CDS & 58,53 & $186-201$ & $\begin{array}{l}\text { ethylene-responsive transcription factor } \\
\text { ERF113 isoform X2 [Sesamum indicum]; } \\
\text { XP_011086885.1 }\end{array}$ \\
\hline GDUL01026627 & $\begin{array}{l}\text { OeUP-06: *TGAAGTTCCAAGCAGTCCAA } \\
\text { R: CTCTTTCTTCAACAGTGGCTGA }\end{array}$ & (TTC)11 & CDS & 50,45 & $141-163$ & $\begin{array}{l}\text { cyclin-D3-2-like [Nicotiana sylvestris]; } \\
\text { XP_009769846.1 }\end{array}$ \\
\hline GDUL01021889 & $\begin{array}{l}\text { FeUP-07 *TATTGATCACACGGGAATCG } \\
\text { R: AACACAATCCAATCCAAGCTG }\end{array}$ & $(\mathrm{GA}) 11$ & 3' UTR & 50,45 & $207-235$ & $\begin{array}{l}\text { phosphatidylinositol transporter, putative [Ricinus } \\
\text { communis]; XP_002512943.1 }\end{array}$ \\
\hline GDUL01011676 & $\begin{array}{c}\text { OeUP-08: *AACGCTCAAGTTCGGTGTTC } \\
\text { R: CCAATCCGGTGGCATGATG }\end{array}$ & $(\mathrm{TGC}) 7$ & 3' UTR & 50,45 & $196-208$ & $\begin{array}{l}\text { mediator of RNA polymerase II transcription } \\
\text { subunit } 8 \text { [Fragaria vesca subsp. vesca]; } \\
\text { XP_004296540.1 }\end{array}$ \\
\hline GDUL01009850 & $\begin{array}{l}\text { 0eUP-09 F: }{ }^{*} \text { TGCTTAGAGCCAGAGGACGA } \\
\text { R: CCCAAAATCCATTTTACACCA }\end{array}$ & $(\mathrm{TGA}) 8$ & CDS & 58,53 & $141-156$ & $\begin{array}{l}\text { hypothetical protein M569_16005, partial [Genli- } \\
\text { sea aurea]; EPS58808.1 }\end{array}$ \\
\hline GDUL01002589 & $\begin{array}{l}\text { OeUP-10: *ACCGGAGCTGAGAATATCCA } \\
\text { R: TCTTCCACTGCCACTACTCG }\end{array}$ & (TCG)7 & CDS & 58,53 & $212-218$ & $\begin{array}{l}\text { zinc finger CCCH domain-containing protein 11- } \\
\text { like [Sesamum indicum]; XP_011077389.1 }\end{array}$ \\
\hline GDUL01041395 & $\begin{array}{l}\text { OeUP-11 } \\
\text { R: *ATAGATAACCACCAGCATTACCATTGCATCA }\end{array}$ & $(\mathrm{TGG}) 7$ & 3' UTR & 53,48 & $137-165$ & $\begin{array}{l}\text { abscisic stress-ripening protein 3-like isoform X1 } \\
\text { [Populus euphratica]; XP_011044239.1 }\end{array}$ \\
\hline GDUL01003880 & $\begin{array}{l}\text { OeUP-12 F: *GAGCTGCTGGGATAGGACTG } \\
\text { R: CTCGATCCCTCACGTTACAAG }\end{array}$ & (TGTA)6 & 3' UTR & 50,45 & $140-151$ & $\begin{array}{l}\text { proteasome-associated protein ECM29 homolog } \\
\text { isoform X2 [Cucumis melo]; XP_008449628.1 }\end{array}$ \\
\hline GDUL01008284 & $\begin{array}{l}\text { OeUP-13 F: *CAATAACCCCATCAGCAGCC } \\
\text { R: AGTCCATTAAAACCTTGCAGTCA }\end{array}$ & (TATG)7 & 3' UTR & 53,48 & $162-174$ & $\begin{array}{l}\text { DDB1-and CUL4-associated factor } 8 \text { [Sesamum } \\
\text { indicum]; XP_011089371.1 }\end{array}$ \\
\hline GDUL01006921 & $\begin{array}{l}\text { OeUP-14 F: *TTCCCTTTCGCTTGAAGGTA } \\
\text { R: GTGTGGATGGCGCTTTAGTC }\end{array}$ & $(\mathrm{AC}) 11$ & 5' UTR & 55,50 & $153-165$ & $\begin{array}{l}\text { predicted protein [Phaeodactylum tricornutum } \\
\text { CCAP 1055/1]; XP_002185470.1 }\end{array}$ \\
\hline GDUL01000672 & $\begin{array}{c}\text { OeUP-15 F: *TCTGGGGTTGCTAATTGTAG } \\
\text { R: TTTCCTTCATCATAAAGCTT }\end{array}$ & (GTG)7 & 3' UTR & 58,53 & $184-190$ & $\begin{array}{l}\text { enhancer of mRNA-decapping protein 4-like } \\
\text { [Nelumbo nucifera]; XP_010279236.1 }\end{array}$ \\
\hline GDUL01011852 & 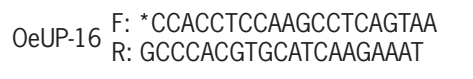 & (TC)10 & 5' UTR & 60,55 & $234-258$ & $\begin{array}{l}\text { growth regulator-like protein [Medicago trun- } \\
\text { catula]; XP_003601898.2 }\end{array}$ \\
\hline GDUL01008290 & $\begin{array}{l}\text { OeUP-17 F: *TCTGGCCTGAGACCTTAAACA } \\
\text { R: CGTGGAGTAATTTCATTGGATG }\end{array}$ & $(\mathrm{GA}) 11$ & 5' UTR & 50,45 & $158-167$ & $\begin{array}{l}\text { Embryo sac development arrest 6, putative } \\
\text { [Theobroma cacao]; XP_007035194.1 }\end{array}$ \\
\hline GDUL01002948 & $\begin{array}{l}\text { OeUP-18 } \\
\text { R: *TACCCGTTTCCCTTCACTTG } \\
\text { R: AGCTGTGACCCTACAATGG }\end{array}$ & $(\mathrm{TCC}) 9$ & CDS & 50,45 & $177-200$ & $\begin{array}{l}\text { disease resistance response protein 206-like } \\
\text { [Glycine max]; XP_003525358.1 }\end{array}$ \\
\hline GDUL01011678 & $\begin{array}{l}\text { OeUP-19 F: *TACAATTCATCTGCGGCATC } \\
\text { R: GGCTTCGATTGATTTGCTGT }\end{array}$ & (GA)10 & 3' UTR & 58,53 & $175-183$ & $\begin{array}{l}\text { E3 ubiquitin-protein ligase CIP8 [Erythranthe gut- } \\
\text { tatus]; XP_012839188.1 }\end{array}$ \\
\hline GDUL01012584 & $\begin{aligned} \text { OeUP-20 F: *ACCAACCGAGCAAAGCAAAA } & \text { R: TTGCCATCGTCTGAGAGTGT }\end{aligned}$ & (TTC)7 & 5' UTR & 50,45 & $191-212$ & $\begin{array}{l}\text { hypothetical protein CICLE_v10016930mg } \\
\text { [Citrus clementina]; XP_006445829.1 }\end{array}$ \\
\hline GDUL01010877 & 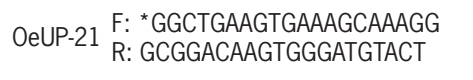 & $(\mathrm{AC}) 15$ & 3' UTR & 55,50 & $190-205$ & $\begin{array}{l}\text { intersectin-1 [Nicotiana tomentosiformis]; } \\
\text { XP_009631701.1 }\end{array}$ \\
\hline GDUL01019797 & $\begin{array}{l}\text { OeUP-22 F: *TAGATTCAAAGGGCGGCTAA } \\
\text { R: GAGGATCTATGGCATCGTCA }\end{array}$ & (GA)13 & 5' UTR & 50,45 & $153-184$ & $\begin{array}{l}\text { MIP18 family protein At1g68310-like [Solanum } \\
\text { tuberosum]; XP_006366991.1 }\end{array}$ \\
\hline GDUL01016632 & $\begin{array}{l}\text { OeUP-23 F: *CAATTGCTGCTGCTGACTTC } \\
\text { R: CATTCAGTTCCTCCCTTCCA }\end{array}$ & $(A G A) 7$ & CDS & 53,48 & $194-197$ & $\begin{array}{l}\text { senescence-associated family protein [Populus } \\
\text { trichocarpa]; XP_006372581.1 }\end{array}$ \\
\hline GDUL01018333 & $\begin{array}{l}\text { OeUP-24 F: *ACCAGATGAAAACTCGTGATCT } \\
\text { R: ATACGTTCGTCTAGCAGCCA }\end{array}$ & (CAG)7 & 5' UTR & 53,48 & $185-200$ & $\begin{array}{l}\text { UBP1-associated proteins 1C [Vitis vinifera]; } \\
\text { XP_002280767.1 }\end{array}$ \\
\hline
\end{tabular}


Table 1 - Continuation.

\begin{tabular}{|c|c|c|c|c|c|c|}
\hline GDUL01023602 & $\begin{array}{l}\text { OeUP-25 F: *AAACATTTTGCACCATTCCA } \\
\text { R: TCAATTGGCTTCTTCCCTCA }\end{array}$ & (ATC)7 & 5' UTR & 55,50 & $171-178$ & $\begin{array}{l}\text { poly(ADP-ribose) glycohydrolase } 1 \text { isoform X1 } \\
\text { [Vitis vinifera]; XP_002280371.1 }\end{array}$ \\
\hline GDUL01004307 & $\begin{array}{l}\text { OeUP-26 F: *AGGAAGTAGCAGCACCAGTT } \\
\text { R: TCCCTCCTTTCCTGGCTTTT }\end{array}$ & (GAT)7 & 3' UTR & 53,48 & $177-204$ & $\begin{array}{l}60 S \text { ribosomal protein L36-2-like [Gossypium } \\
\text { raimondii]; XP_012482487.1 }\end{array}$ \\
\hline GDUL01015903 & $\begin{array}{l}\text { OeUP-27 F: *GGCTCACCCTTTCAGTTTCA } \\
\text { R: CAGCCCATGAATCAGCATCC }\end{array}$ & (TTC)9 & 5' UTR & 55,50 & $159-181$ & $\begin{array}{l}\text { protein dehydration-induced } 19 \text { homolog } \\
\text { 3-like isoform X1 [Erythranthe guttatus]; } \\
\text { XP_012837687.1 }\end{array}$ \\
\hline GDUL01057837 & $\begin{array}{l}\text { OeUP-28 F:TTTTAGTTCAAACTACATAGGAACGTA } \\
\text { R: *CAAGCTGGTGCCTTATGGTT }\end{array}$ & (AG)10 & 5' UTR & 50,45 & $152-184$ & $\begin{array}{l}\text { glutaredoxin-C9-like [Nicotiana sylvestris]; } \\
\text { XP_009767693.1 }\end{array}$ \\
\hline GDUL01000197 & $\begin{array}{l}\text { OeUP-29 F: *GTCCTGCCTTCAAGATCCAC } \\
\text { R: GCGGTGGCCTGAGATACTTA }\end{array}$ & (CA)10 & CDS & 55,50 & $205-215$ & $\begin{array}{l}\text { protein yippee-like At5g53940-like [Solanum } \\
\text { tuberosum]; XP_006358970.1 }\end{array}$ \\
\hline GDUL01007444 & $\begin{array}{l}\text { OeUP-30 F:GAGTCACACTCGTCCCTACCA } \\
\text { R: *GAATCGCGCGTGTAACATTT }\end{array}$ & $(\mathrm{AC}) 11$ & 5' UTR & 55,50 & $190-196$ & $\begin{array}{l}\text { autophagy-related protein 8i-like [Solanum } \\
\text { tuberosum]; NP_001275429.1 }\end{array}$ \\
\hline GDUL01003407 & $\begin{array}{l}\text { OeUP-31 F: * }{ }^{*} \text { RATTTTCCGACCAACTGATGAA } \\
\text { R: TCTTCTTGCGGTTAGGTTGC }\end{array}$ & $(\mathrm{ACC}) 8$ & 5' UTR & 60,55 & 203-212 & $\begin{array}{l}\text { E3 ubiquitin-protein ligase UBR2 [Sesamum } \\
\text { indicum]; XP_011077478.1 }\end{array}$ \\
\hline GDUL01001906 & $\begin{array}{c}\text { OeUP-32 F: *ACGAACCGTGGCAGTACATT } \\
\text { R: CCTTCCCTTTGCCTGTATGA }\end{array}$ & $(A G A) 7$ & 5' UTR & 55,50 & $171-188$ & $\begin{array}{l}\text { Rubredoxin-like superfamily protein [Theobroma } \\
\text { cacao]; XP_007038169.1 }\end{array}$ \\
\hline GDUL01002038 & $\begin{array}{c}\text { OeUP-33 F: *ACGTCAACCATGCTTCAACC } \\
\text { R: TCAGTACCGAAGCAGCCATA }\end{array}$ & (TCA)7 & 3' UTR & 53,48 & $220-234$ & $\begin{array}{l}\text { Cold-regulated 413-plasma membrane } 2 \text { isoform } \\
1 \text { [Theobroma cacao]; XP_007047094.1 }\end{array}$ \\
\hline GDUL01058412 & $\begin{array}{l}\text { OeUP-34 F: CCTTCATAGGCAAAATGGTCA } \\
\text { R: *CACCAAAGCATTTGCAGAGA }\end{array}$ & $(\mathrm{GA}) 12$ & 3' UTR & 55,50 & $193-226$ & $\begin{array}{l}\text { xyloglucan endotransglucosylase/hydrolase } 3 \\
\text { [Actinidia eriantha]; ACD03213.1 }\end{array}$ \\
\hline GDUL01009565 & $\begin{array}{l}\text { OeUP-35 F: *GGTGGGGTCTGAATATATGTCA } \\
\text { R: GGCAATGACTTTACGACAAGA }\end{array}$ & (ATT)7 & 3' UTR & 58,53 & $225-231$ & $\begin{array}{l}\text { hypothetical protein MIMGU_mgvla003502mg } \\
\text { [Erythranthe guttata]; EYU41540.1 }\end{array}$ \\
\hline GDUL01055178 & $\begin{array}{l}\text { OeUP-36 F: *TGCTTTCTCATTTTCACCACCA } \\
\text { R: AGACTGCACCACCATCATCA }\end{array}$ & (GTC)7 & 3' UTR & 58,53 & $142-154$ & $\begin{array}{l}\text { ADNP homeobox protein } 2 \text { Tupaia chinensis]; } \\
\text { XP_006165827.1 }\end{array}$ \\
\hline GDUL01015452 & $\begin{array}{l}\text { OeUP-37 F: *AATGGCTGCGAAATGAGAAG } \\
\text { R: CAAAGATTTCAATTTTGTTCTTGGT }\end{array}$ & (TTC)9 & CDS & 50,45 & $148-188$ & $\begin{array}{l}\text { pentatricopeptide repeat-containing protein, puta- } \\
\text { tive [Ricinus communis]; XP_002509440.1 }\end{array}$ \\
\hline GDUL01057556 & $\begin{array}{l}\text { OeUP-38 F: *AGAACCACCTCAACCACCAA } \\
\text { R: GTCGTCTGTTGTTGGCGATA }\end{array}$ & (CAT)7 & CDS & 53,48 & $150-167$ & $\begin{array}{l}\text { Serine/threonine-protein kinase HuHRK1 } \\
\text { [Hanseniaspora uvarum DSM 2768]; } \\
\text { KKA02402.1 }\end{array}$ \\
\hline GDUL01033407 & $\begin{array}{l}\text { OeUP-39 F: }{ }^{*} \text { TCTCGGTTCCTTCCATTCCA } \\
\text { R: GCGAAACAGAAATACATACAAGG }\end{array}$ & $(A G) 10$ & 3' UTR & 50,45 & 193-209 & $\begin{array}{l}\text { hypothetical protein L484_004798 [Morus } \\
\text { notabilis]; XP_010099372.1 }\end{array}$ \\
\hline GDUL01030115 & $\begin{array}{ll}\text { OeUP-40 } & \text { F: *ACCCAGTAGCTTAAATCTGT } \\
& \text { R: ACGACGATGCGATTTCTTCA }\end{array}$ & (TCT)7 & 3' UTR & 53,48 & $179-197$ & $\begin{array}{l}\text { High chlorophyll fluorescence phenotype } \\
173 \text { protein isoform } 1 \text { [Theobroma cacao]; } \\
\text { XP_007023557.1 }\end{array}$ \\
\hline GDUL01029326 & $\begin{array}{ll}\text { OeUP-41 } & \text { F: *CGGTGACCCTTTAGCCTGTA } \\
& \text { R: ATCAGAGGCTTCGAGGAGGT }\end{array}$ & (AAG)7 & CDS & 50,45 & $160-163$ & $\begin{array}{l}\text { lisH domain-containing protein C1711.05-like } \\
\text { [Citrus sinensis]; XP_006482262 }\end{array}$ \\
\hline GDUL01034902 & $\begin{array}{ll}\text { OeUP-42 } & \text { F: *CGATTTCAAAGGGAATCGAA } \\
\text { R: ACCCGCAATTTACCATGAAA }\end{array}$ & (CTG)8 & CDS & 58,53 & $162-183$ & $\begin{array}{l}\text { vacuolar protein sorting-associated protein } \\
8 \text { homolog isoform X1 [Vitis vinifera]; } \\
\text { XP_002267626.3 }\end{array}$ \\
\hline GDUL01048166 & $\begin{array}{ll}\text { OeUP-43 F: AAAATCAAACACAACTTGGCAAT } \\
\text { R: *CTTCCAATCTGAAAATGCACA }\end{array}$ & (TTC)7 & CDS & 50,45 & $195-231$ & $\begin{array}{l}\text { TrbL/NirB6 plasmid conjugal transfer protein } \\
\text { [Neisseria wadsworthii]; ZP_08940344.1 }\end{array}$ \\
\hline GDUL01050680 & $\begin{array}{ll}\text { OeUP-44 } & \text { F: *TCAGGTTCTGGCAAGGATTC } \\
\text { R:TTCATCCCTTCTGCTGCTCT }\end{array}$ & (GCT)7 & CDS & 58,53 & $149-155$ & $\begin{array}{l}\text { protein MEI2-like } 4 \text { isoform X3 [Vitis vinifera]; } \\
\text { XP_010657314.1 }\end{array}$ \\
\hline KU709832 & $\begin{array}{ll}\text { OeUP-45: } & \text { *GGCCAATCAATGAACCAGCA } \\
& \text { R: AGGGCTTGATTCTAAGCTCA }\end{array}$ & $(A A G) 7$ & CDS & 50,45 & $145-168$ & $\begin{array}{l}\text { flavonoid-related R2R3 MYB 4a repressor } \\
\text { transcription factor [Vitis vinifera]; } \\
\text { NP_001268129.1 }\end{array}$ \\
\hline GDUL01050819 & $\begin{array}{ll}\text { OeUP-46 } & \text { F: *TCTTCCTGGTATTTGTTTCGGT } \\
& \text { R: ACAAGAGTATATATGCCACACA }\end{array}$ & $(\mathrm{AAT}) 7$ & CDS & 58,53 & $201-204$ & $\begin{array}{l}\text { hypothetical protein [Methylococcaceae } \\
\text { bacterium 73a]; WP_045223293.1 }\end{array}$ \\
\hline
\end{tabular}

*Primers elongated for the universal M13 (-21) sequence (5'-TGTAAAACGACGGCCAGT-3') at the 5' ends.

\section{Putative functional annotation of the transcripts containing SSRs}

All ESTs containing SSRs were further used for functional annotation with Blast2GO online application (https:/www.blast2go.com) in order to assign the sequences to the following functional Gene Ontology (GO) categories: molecular function, biological process, and cellular component. In addition, Blast2GO was used to assign Kyoto Encyclopedia of Genes and Genomes (KEGG) maps and an enzyme classification number (EC) (Kanehisa and Goto, 2000). For developed EST-SSRs, the position of the SSR motif in the gene was performed, that is, the SSR was located in coding sequence (CDS), 3' untranslated region (3' UTR) or $5^{\prime}$ untranslated region (5' UTRs). 


\section{Results}

Characterization of EST-SSRs and primer designing A total of 98,924 ESTs $(36.8 \mathrm{Mb})$ from the olive transcriptome assembly (Rešetič et al., 2013) were examined with the MISA tool for microsatellite identification. Of 98,924 ESTs, 923 sequences contained 984 microsatellites. On average, one microsatellite was found in every $37.4 \mathrm{~kb}$ of olive ESTs. Of 923 SSR-containing ESTs, $874(95 \%)$ ESTs contained only one SSR locus, while 49 (5\%) contained more than one SSR locus. Furthermore, $4 \%$ of SSR loci (34 of all identified SSRs) were present in the compound formation.

The most abundant SSRs in olive were dinucleotide repeats $(36 \%)$, followed by tri- $(33 \%)$, hexa- (21 $\%)$ and penta-nucleotide $(9 \%)$ repeat units. The most frequent dinucleotide motif was GA/TC (37\%), followed by AG/CT $(30 \%)$ and AC/GT (17\%). Among trinucleotides, GAA/TTC was the most abundant (16\%). Motifs AGA/TCT and AAG/CTT occurred at $12 \%$ and $11 \%$, respectively.

Of 984 EST-SSRs, 551 EST-SSRs possessing microsatellite sequences longer than $20 \mathrm{bp}$ were further reviewed by the Tablet program. A total of 343 EST-SSRs had flanking regions longer than 30 bases and they were not located in the overlapping site within EST contig. Among these, only 197 EST-SSRs containing di-, tri-, tetra-nucleotides and compound SSRs were selected and blasted against the NCBI nr protein database. A total of 119 successfully annotated EST-SSRs were subjected to primer designing. Finally, 110 EST-SSR primer pairs were designed for further amplification.

\section{Marker validation}

Novel markers were tested for genotyping in a set of eight varieties of olive. Of 110 EST-SSR developed primers, only one (1\%) was monomorphic and 46 (42\%) demonstrated polymorphism with two or more alleles that could be detected and reliably scored. Among the others, $63(57 \%)$ loci fell into the following groups according to Chen et al. (2014): (1) "no peak" (45\%), (2) "weak peak" $(11 \%)$ and "multiple peaks" $(2 \%)$, based on the peak detection during the genotyping procedure. These 46 newly developed EST-SSRs contained trinucleotides (67\%), dinucleotides (26\%), two tetranucleotide repeats and one compound SSR (Table 1).

\section{Polymorphism and genetic diversity analysis}

All 46 developed EST-SSRs were used to assess the polymorphism and genetic diversity of 24 olive varieties. All loci were successfully amplified and 205 different alleles were detected. The number of amplified alleles at each locus varied from two (OeUP-10, OeUP-12, OeUP17, OeUP-23, OeUP-25, OeUP-41, OeUP-46) to eight (OeUP-22 and OeUP-34). The average number of alleles per locus was 4.5 and the average number of effective alleles was 3.13. $\mathrm{H}_{\mathrm{e}}$ ranged between 0.042 (OeUP-12) and 0.869 (OeUP-22), with an average of 0.604 . The highest
$\mathrm{H}_{\mathrm{o}}$ (1.000) was found at locus OeUP-22, and the lowest (0.042) was observed at two loci (OeUP-12 and OeUP43). PI value varied among loci in a range from 0.076 (OeUP-22) to 0.922 (OeUP-12), while the common PI value calculated for all loci was $2.10 \times 10^{-24}$. PIC values were in a range from 0.040 (OeUP-12) and 0.833 (OeUP$22)$, with a mean value 0.539 . Based on the calculated PIC, 30 newly developed EST-SSRs were classified as informative markers (PIC > 0.5) and nine as suitable markers for gene mapping (PIC > 0.7) (Table 2).

Of 46 EST-SSR loci, 11 loci showed deviation from the HWE (Table 2). Most deviations can be assigned to the excess of one class and, more rarely, to two classes of homozygotes in the analyzed loci as well as to an excess of one combination of identical alleles observed in a larger set of olive varieties. In nine loci, $\mathrm{H}_{\mathrm{o}}$ was lower than $\mathrm{H}_{\mathrm{e}}$, and in six loci, the estimated frequencies of null alleles were higher than 0.2 , indicating the upper boundary under which the microsatellite null alleles are uncommon to rare (Dakin and Avise, 2004).

Among 205 alleles detected, 29 were specific to different olive varieties. The allelic polymorphisms allowed discriminating all analyzed varieties. The AMaCAID computer program was used to calculate the minimum number of markers required to distinguish all observed genotypes. All 24 olive genotypes could be differentiated by only two loci (OeUP-04 and OeUP-14).

The number of different microsatellite genotypes observed per locus ranged from two (OeUP-12, OeUP17, OeUP-23, and OeUP-46) to 14 (OeUP-01 and OeUP22), with 311 different genotypes, revealing $49 \%$ of all possible genotypes (641). Altogether, 114 unique genotypes were observed. The first three loci with the highest number of unique genotypes were OeUP-01 (9), OeUP16 (8) and OeUP-34 (7).

The Jaccard's similarity coefficient was used to calculate the genetic distances among pairwise combinations in a set of 24 olive varieties from the Slovenian olive collection. The highest genetic similarity value (0.72) was observed among the varieties "Leccino" and "Zelenjak". The average similarity coefficient was relatively low (0.39). A dendrogram (Figure 1) was constructed from genetic similarity data and clusters were tested for associations. The cophenetic coefficient was relatively high $(0.84)$ and indicated a good fit of the original data to the clustering.

Olive varieties clustered into related groups in the microsatellite dendrogram. The first cluster, which showed higher genetic similarities with respect to the other groups, contained all Tuscan varieties for oil use ("Leccino", "Leccio del corno", "Pendolino", "Leccione, "Maurino", "Frantio"). This cluster also contained the Italian variety "Coratina" as well as "Athena' and "Zelenjak", which were probably brought from central Italy to the northern Adriatic coast as shown in a previous study (Bandelj et al., 2004). "Črnica" and "Štorta" were also close to the Tuscan group, which are known as Slovenian varieties for oil production and table use, re- 
spectively. "Ascolana tenera", "Grignan", "Buga", "Samo" and "Oblica" were well-defined groups. These varieties shared more than $60 \%$ of alleles and two of them ("Samo" and "Buga") represent the local Slovenian germ- plasm. The varieties "Arbequina", "Cipressino", "Istrska belica" and "Moraiolo" were placed on the dendrogram at lower similarity values. For these varieties, two or more unique alleles were found.

Table 2 - Parameters of genetic variability of each expressed sequence tag-derived simple sequence repeat (EST-SSR) obtained among 24 olive varieties. Observed $\left(H_{0}\right)$ and expected $\left(H_{e}\right)$ heterozygosity, number of alleles $(n)$, effective number of alleles $\left(n_{e}\right)$, polymorphic information content (PIC), probability of identity (PI), probability for deviation from Hardy-Weinberg equilibrium ( $p$-value), estimated frequency of null alleles (F(null)).

\begin{tabular}{|c|c|c|c|c|c|c|c|c|}
\hline Locus & $\mathrm{H}_{0}$ & $\mathrm{H}_{\mathrm{e}}$ & $n$ & $\mathrm{n}_{\mathrm{e}}$ & PIC & $\mathrm{PI}$ & $p$-value & F(null) \\
\hline OeUP-01 & 0.833 & 0.811 & 6 & 5.29 & 0.764 & 0.132 & $0.5507^{\mathrm{NS}}$ & -0.0235 \\
\hline OeUP-02 & 0.833 & 0.748 & 6 & 3.97 & 0.686 & 0.212 & $0.3597^{\mathrm{NS}}$ & -0.0689 \\
\hline OeUP-03 & 0.958 & 0.563 & 3 & 2.29 & 0.454 & 0.494 & $0.0002^{* \star *}$ & -0.2870 \\
\hline OeUP-04 & 0.875 & 0.824 & 7 & 5.68 & 0.780 & 0.120 & $0.4429^{\text {NS }}$ & -0.0376 \\
\hline OeUP-05 & 0.583 & 0.593 & 4 & 2.46 & 0.539 & 0.300 & $0.0515^{\mathrm{NS}}$ & +0.0299 \\
\hline OeUP-06 & 0.958 & 0.785 & 6 & 4.65 & 0.730 & 0.169 & $0.2551^{\mathrm{NS}}$ & -0.1171 \\
\hline OeUP-07 & 0.208 & 0.696 & 7 & 3.29 & 0.638 & 0.231 & $0.0000^{* * *}$ & +0.5433 \\
\hline OeUP-08 & 0.708 & 0.629 & 4 & 2.70 & 0.571 & 0.283 & $0.5822^{\mathrm{NS}}$ & -0.0636 \\
\hline OeUP-09 & 0.958 & 0.792 & 5 & 4.81 & 0.740 & 0.157 & $0.2284^{\text {NS }}$ & -0.1079 \\
\hline OeUP-10 & 0.583 & 0.507 & 2 & 2.03 & 0.373 & 0.623 & $0.4516^{\mathrm{NS}}$ & -0.0804 \\
\hline OeUP-11 & 0.875 & 0.717 & 7 & 3.53 & 0.654 & 0.233 & $0.3174^{\mathrm{NS}}$ & -0.1197 \\
\hline OeUP-12 & 0.042 & 0.042 & 2 & 1.04 & 0.040 & 0.922 & $1.0000^{\mathrm{NS}}$ & -0.0036 \\
\hline OeUP-13 & 0.667 & 0.637 & 3 & 2.75 & 0.553 & 0.353 & $0.5146^{\mathrm{NS}}$ & -0.0270 \\
\hline OeUP-14 & 0.500 & 0.684 & 4 & 3.16 & 0.609 & 0.289 & $0.3739^{\text {NS }}$ & +0.1570 \\
\hline OeUP-15 & 0.208 & 0.407 & 3 & 1.69 & 0.354 & 0.495 & $0.0001^{* * *}$ & +0.3294 \\
\hline OeUP-16 & 0.958 & 0.792 & 6 & 4.81 & 0.741 & 0.152 & $0.7158^{\mathrm{NS}}$ & -0.1096 \\
\hline OeUP-17 & 0.167 & 0.156 & 2 & 1.18 & 0.141 & 0.753 & $0.7025^{\mathrm{NS}}$ & -0.0358 \\
\hline OeUP-18 & 0.875 & 0.737 & 4 & 3.80 & 0.668 & 0.238 & $0.4240^{\mathrm{NS}}$ & -0.0977 \\
\hline OeUP-19 & 0.875 & 0.658 & 4 & 2.92 & 0.594 & 0.276 & $0.0819^{\mathrm{NS}}$ & -0.1783 \\
\hline OeUP-20 & 0.875 & 0.738 & 6 & 3.82 & 0.673 & 0.226 & $0.1835^{\mathrm{NS}}$ & -0.1068 \\
\hline OeUP-21 & 0.792 & 0.701 & 4 & 3.34 & 0.628 & 0.274 & $0.4591^{\text {NS }}$ & -0.0670 \\
\hline OeUP-22 & 1.000 & 0.869 & 8 & 7.63 & 0.833 & 0.076 & $0.3407^{\mathrm{NS}}$ & -0.0823 \\
\hline OeUP-23 & 0.500 & 0.383 & 2 & 1.62 & 0.305 & 0.602 & $0.1205^{\text {NS }}$ & -0.1423 \\
\hline OeUP-24 & 0.958 & 0.780 & 6 & 4.55 & 0.731 & 0.155 & $0.0586^{\mathrm{NS}}$ & -0.1223 \\
\hline OeUP-25 & 0.125 & 0.488 & 2 & 1.95 & 0.364 & 0.615 & $0.0001^{* * *}$ & +0.5856 \\
\hline OeUP-26 & 0.875 & 0.667 & 5 & 3.00 & 0.589 & 0.313 & $0.4038^{\text {NS }}$ & -0.1633 \\
\hline OeUP-27 & 0.958 & 0.664 & 5 & 2.98 & 0.585 & 0.318 & $0.0466^{*}$ & -0.2089 \\
\hline OeUP-28 & 0.333 & 0.489 & 5 & 1.96 & 0.433 & 0.409 & $0.0002^{* * *}$ & +0.1823 \\
\hline OeUP-29 & 0.917 & 0.660 & 4 & 2.94 & 0.577 & 0.334 & $0.0581^{\mathrm{NS}}$ & -0.1840 \\
\hline OeUP-30 & 0.792 & 0.611 & 3 & 2.57 & 0.514 & 0.414 & $0.2565^{\mathrm{NS}}$ & -0.1470 \\
\hline OeUP-31 & 0.417 & 0.391 & 4 & 1.64 & 0.355 & 0.464 & $0.9763^{\mathrm{NS}}$ & -0.0349 \\
\hline OeUP-32 & 0.625 & 0.595 & 3 & 2.47 & 0.514 & 0.379 & $0.4851^{\text {NS }}$ & -0.0194 \\
\hline OeUP-33 & 0.167 & 0.515 & 4 & 2.06 & 0.458 & 0.386 & $0.0000^{* * *}$ & +0.4955 \\
\hline OeUP-34 & 0.958 & 0.860 & 8 & 7.14 & 0.823 & 0.083 & $0.0619^{\mathrm{NS}}$ & -0.0692 \\
\hline OeUP-35 & 0.542 & 0.414 & 3 & 1.71 & 0.338 & 0.554 & $0.3892^{\mathrm{NS}}$ & -0.1522 \\
\hline OeUP-36 & 0.292 & 0.263 & 3 & 1.36 & 0.239 & 0.606 & $0.8989^{\text {NS }}$ & -0.0723 \\
\hline OeUP-37 & 0.958 & 0.848 & 7 & 6.58 & 0.808 & 0.095 & $0.3292^{N S}$ & -0.0725 \\
\hline OeUP-38 & 0.667 & 0.639 & 5 & 2.77 & 0.553 & 0.359 & $0.0000^{* * *}$ & -0.0610 \\
\hline OeUP-39 & 0.833 & 0.735 & 6 & 3.77 & 0.669 & 0.230 & $0.1459^{\text {NS }}$ & -0.0780 \\
\hline OeUP-40 & 0.667 & 0.655 & 4 & 2.90 & 0.590 & 0.283 & $0.4966^{\mathrm{NS}}$ & -0.0229 \\
\hline OeUP-41 & 0.292 & 0.510 & 2 & 2.04 & 0.375 & 0.625 & 0.0322 & +0.2623 \\
\hline OeUP-42 & 0.542 & 0.576 & 6 & 2.36 & 0.528 & 0.299 & $0.5137^{\mathrm{NS}}$ & +0.0596 \\
\hline OeUP-43 & 0.042 & 0.299 & 4 & 1.43 & 0.274 & 0.555 & $0.0000^{* * *}$ & +0.7658 \\
\hline OeUP-44 & 0.583 & 0.504 & 3 & 2.02 & 0.391 & 0.565 & $0.4816^{\mathrm{NS}}$ & -0.0788 \\
\hline OeUP-45 & 0.875 & 0.730 & 6 & 3.70 & 0.671 & 0.213 & $0.5332^{\mathrm{NS}}$ & -0.1208 \\
\hline OeUP-46 & 0.625 & 0.439 & 2 & 1.78 & 0.337 & 0.602 & 0.0324 & -0.1850 \\
\hline Average & 0.649 & 0.604 & 4.5 & 3.13 & 0.539 & $2.10 \times 10^{-24 a}$ & - & - \\
\hline
\end{tabular}

aProduct of PI values for 46 EST-SSR loci; NS = not significant $p>0.05$; * Statistically significant difference $p<0.05$; ** Statistically significant difference $p<0.001$. 


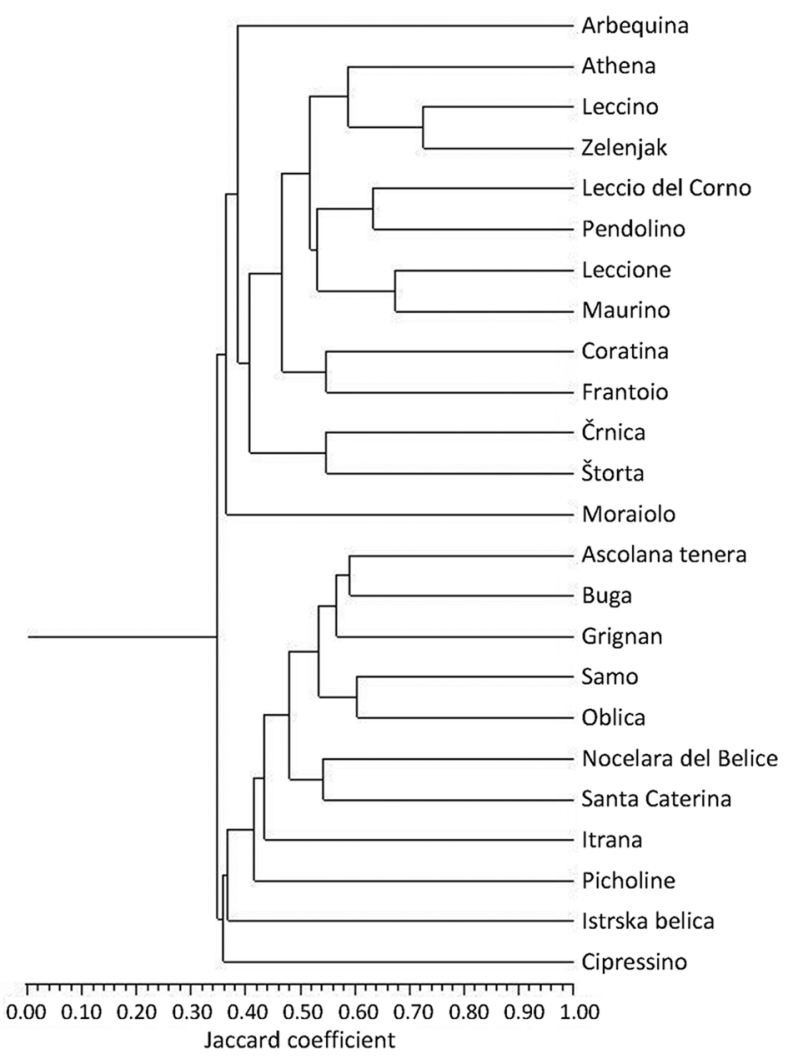

Figure 1 - Phylogenetic tree of 24 olive varieties based on the Jaccard's coefficient and unweighted pair-group method with arithmetic averages (UPGMA).

\section{Functional annotation of SSR-containing tran- scripts}

In order to assign the putative function of all 923 ESTs containing SSRs, a functional annotation was carried out using the Blast2GO software. A total of 247 ESTs (27\%) were detected as having homology with known proteins, 138 (15\%) were homologous to expressed, hypothetical/unknown/unnamed proteins, while 538 (58 \%) SSR-containing sequences showed no significant $(\mathrm{E}<1 \mathrm{e}-10)$ hits in the BLASTX analysis. During the BLASTing step, ESTs (109) were aligned to Sesamum indicum L., while only six sequences were in correspondence to the Olea europaea L. database.

The following gene ontology categories, molecular function, biological process, and cellular component, were then assigned to 247 ESTs having homology with known proteins (Figure 2). A total of 265 terms were allocated to the molecular function, 671 under biological process, and 233 under the cellular component. The most abundant ESTs were involved in metabolic (22\%), cellular $(21 \%)$ and single-organism processes (18 \%) under the biological process category. According to the molecular function category, most sequences showed functions related to binding $(45 \%)$, catalytic activity (39 $\%)$ and transporter activity $(6 \%)$. The classification of the sequences within the binding group of the molecular function category showed that most ESTs fell under ion binding $(34 \%)$ and Adenosine triphosphate (ATP) binding $(20 \%)$. For the cellular component category, $54 \%$ of the sequences were assigned to term intracellular followed by an intrinsic component of the membrane $(14 \%)$ and cell periphery $(9 \%)$. A part of newly developed and annotated EST-SSRs was related to a lipid biosynthetic process (OeUP-16), cellular response to

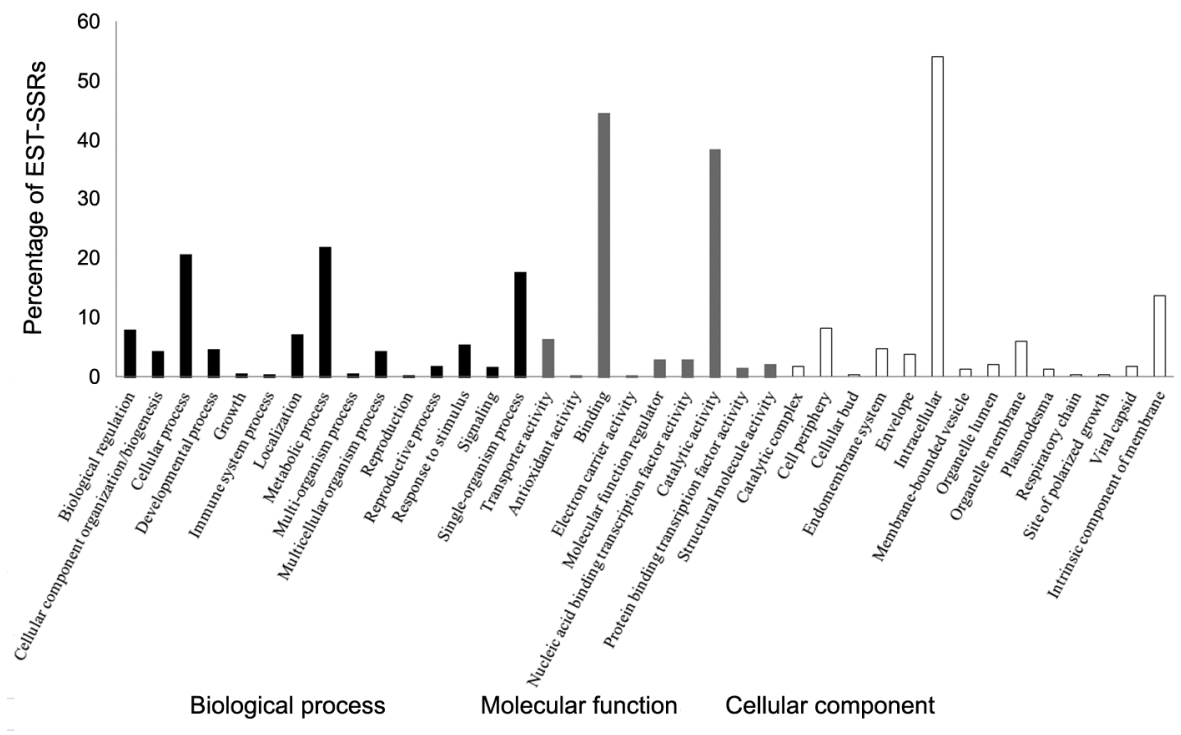

Figure 2 - Functional annotations of 247 expressed sequence tag-derived simple sequence repeats (EST-SSRs) in olives based on the Blast2G0 analysis. 
oxidative stress (OeUP-23 and OeUP-25) and osmotic stress (OeUP-25), with cell transport (OeUP-01, OeUP07, OeUP-30, OeUP-42) and with embryo development (OeUP-09 and OeUP-17).

In addition, KEGG pathway visualization and EC annotations were also done for 247 annotated ESTs. In total, 55 pathways, including numerous cellular metabolic and biosynthesis pathways, were fully represented. Since olive is an important oil crop, the research was focused on ESTs with particular relevance in fruit metabolism. Most ESTs encoded the following enzymes: transferases $(37 \%)$, hydrolases $(33 \%)$ and oxidoreductases (19\%). Specifically, ESTs encoded enzymes for biosynthesis of secondary metabolites and lipids, including fatty acid and steroid biosynthesis, as well as sphingolipid metabolism.

Sequences of 46 polymorphic EST-SSRs were classified according to the position of SSRs within genes, that is, in the $3^{\prime}$ untranslated region ( $\left.3^{\prime} \mathrm{UTR}\right), 5^{\prime}$ untranslated region (5'UTR), and coding region (CDS). More than half of developed EST-SSRs (65\%) were located in UTRs (35\% in $3^{\prime}$ UTRs, $30 \%$ in $5^{\prime}$ UTRs) while others $(35 \%)$ were part of the CDSs (Table 1). Among the repeats identified in UTRs, the predominant were trinucleotides (35\%), followed by di- $(24 \%)$ and tetra-nucleotide repeats $(4 \%)$. Only one compound microsatellite $(2 \%)$ was found in UTRs. The most abundant repeats found in the CDS, where the open reading frame $(\mathrm{ORF})$ was not corrupted by changing the number of the core repeat, were trinucleotides (33\%) and only one dinucleotide $(2 \%)$ was identified.

\section{Discussion}

The improvement of genetic resources of agricultural plants through molecular breeding programs requires efficient molecular markers in combination with linkage maps and genomics (Jiang, 2013). Recently, a vast number of EST datasets have been generated for many crop plants, which have offered an opportunity to identify and develop numerous functional molecular markers linked to genes or traits of interest. Although the olive tree is one of the most important oil crops in the Mediterranean, only a few tested EST-SSRs are available (Adawy et al., 2015; De la Rosa et al., 2013; Essalouh et al., 2014). This fact encouraged the Slovenian team to focus their research on the development of new olive EST-SSRs and provide high quality and informative markers to the research community.

For the development of EST-SSRs, the olive transcriptome from developing fruits of variety "Istrska belica" was used. Transcripts were generated from the predominant local variety in Slovenia, which has special organoleptic properties due to high biophenol content and is also known as one of the oiliest varieties in the region. For SSRs identification, 98,924 ESTs were examined. Microsatellites were found in approximately $1 \%$ of olive ESTs. Considerably higher frequencies were re- ported for other fruit species, including $20 \%$ in citrus fruits (Liu et al., 2013), $18 \%$ in peaches (Vendramin et al., 2007) and $11 \%$ in pomegranates (Jian et al., 2012). These differences in frequency and distribution of ESTSSRs can be attributed to the different criteria used to identify SSRs in the database mining, dataset size and database-mining tools (Varshney et al., 2005). Furthermore, variations in the SSR frequency distribution are taxon-specific (Toth et al., 2000) and may reflect differences in the selection and domestication processes (Zhang et al., 2013).

In this study, dinucleotide SSRs were the most abundant $(36 \%)$, which is in accordance with results reported for sesame (Zhang et al., 2012) and some Rosaceae species (Jung et al., 2005). However, in most other fruit species and crop plants, trinucleotide repeats have been observed at the highest frequency (Jian et al., 2012; Liu et al., 2013). In olive transcriptome data, the most abundant dinucleotide SSR motif was GA/TC, while the motif GAA/TTC was the most common among trinucleotide SSRs, which support findings of Adawy et al. (2015). Furthermore, similar results have also been obtained in studies on citrus fruits (Chen et al., 2006), grapes (Huang et al., 2011), and mangos (Dillon et al., 2014).

After the annotation and assignment of putative functions to olive EST-SSRs, primers were successfully designed for 110 loci. After the optimization of the PCR protocol in a set of eight olive varieties, quality amplicons were obtained for 46 primers developed. The lengths of amplified microsatellites were in the expected range and no deviations were found. All 46 primers were used to test their applicability in a diversity study in a set of 24 olive varieties. All 46 EST-SSRs loci were polymorphic and 205 different alleles were detected, with the average number of 4.5 alleles per locus. Averages for $\mathrm{H}_{\mathrm{o}}$ and $\mathrm{H}_{\mathrm{e}}$ were 0.649 and 0.604 , respectively. Slightly greater results for observed and expected heterozygosity $\left(\mathrm{H}_{\mathrm{o}}\right.$ $=0.769, \mathrm{H}_{\mathrm{e}}=0.705$ ) were obtained in a study, where 19 varieties, also used in this study, were tested with genomic SSRs (Bandelj et al., 2004). The comparable ability of genomic and EST-SSRs in the detection of genetic diversity in olives was also confirmed by De la Rosa et al. (2013). Furthermore, EST-SSRs may actually prove to be superior to genomic SSRs for diversity estimation and transferability (Gupta et al., 2003) and should be even more useful to develop linkage maps or tag agronomical important traits (Huang et al., 2011).

A good discrimination power of new genic markers was demonstrated with the selection of the minimum number of markers needed to distinguish all 24 olive varieties. Two loci (OeUP-04 and OeUP-14) were determined to be sufficient for the unambiguous discrimination of all samples. The high discrimination power of EST-SSRs was also confirmed by a large number of genotypes observed per locus, including unique genotypes, as well as 29 unique alleles detected.

Eleven out of 46 developed EST-SSRs showed a deviation from the HWE. In nine loci, the heterozygos- 
ity observed was lower than expected. Since deviations from the HWE in these loci are prone to bias-likelihood estimates, attention should be paid on account of the analyses of identity and parentage as well as in population studies (Cipriani et al., 2010).

The average PIC value for these markers in the genotypes examined was 0.539, which is much higher than those found in citrus genera (mean $=0.450$ ), pomegranate $($ mean $=0.381)$ and carob trees $($ mean $=$ 0.420) (Jian et al., 2012; La Malfa et al., 2014; Liu et al., 2013). Furthermore, 27 newly developed EST-SSRs were classified as informative markers (OeUP-01, OeUP02, OeUP-04, OeUP-05, OeUP-06, OeUP-08, OeUP-09, OeUP-11, OeUP-13, OeUP-14, OeUP-16, OeUP-18, OeUP-19, OeUP-20, OeUP-21, OeUP-22, OeUP-24, OeUP-26, OeUP-29, OeUP-30, OeUP-32, OeUP-34, OeUP-37, OeUP-39, OeUP-40, OeUP-42, OeUP-45), and are strongly recommended due to the low frequency of null alleles and no deviation observed from HWE. These 27 EST-SSRs can be used for olive diversity and population studies.

Comparing results of the clustering analysis with those obtained by genomic SSRs (Bandelj et al., 2004) revealed a similar distribution of olive varieties in related groups. Common genetic background was confirmed for all Tuscan olive varieties for oil use. The clustering analysis with new genic markers confirmed that Slovenian varieties "Zelenjak" and "Črnica" are closed related with Tuscan olives, as previously noted (Bandelj et al., 2004). Both genomic and functional EST-SSRs also showed that the predominant local variety "Istrska belica" had very low genetic similarities with other varieties. These results confirmed the equal ability and functionality of the use of genic SSRs for studies on genetic relationships in olives.

Alignment and functional annotation of all 923 ESTs containing microsatellite were performed using the Blast2GO tool. A total of 246 ESTs had homology with known proteins and were further annotated against the GO database. Ten newly developed EST-SSRs were associated with lipid biosynthetic process, embryo development, and cellular response to stress, while no GO terms were determined for other markers. However, other sequences $(138,15 \%)$ were homologous to expressed, hypothetical/unknown/unnamed proteins, while 538 (58\%) SSR-containing sequences showed no hits.

A relatively high percentage of EST-SSRs without BLASTX hits could be attributed to the protein database used, while EST-SSRs are frequently positioned in UTR regions. When reviewing the location of 46 EST-SSRs, $65 \%$ EST-SSRs were positioned in UTRs, and only $35 \%$ was located in the coding region. Liu et al. (2013) have also observed higher density of di-, tri- and tetra-nucleotide SSRs in UTRs. It has been noted that untranslated regions of mRNAs have crucial roles in many aspects of gene regulation (Mignone et al., 2002). In a study on rice and Arabidopsis, Fujimori et al. (2003) concluded that microsatellites are located at high frequency in the $5^{\prime}$-flanking region of plant genes and potentially acts as factors to regulate gene expression. In contrast, microsatellite expansion in the 3' UTRs can cause transcription slippage and produce expanded mRNA (Li et al., 2004). An elevated percentage of sequences with no putative function may also only be attributed to specifically evolved gene functions and exclusive characteristics to O. europaea species, as reported by Alagna et al. (2009). The analysis of motifs of microsatellites in olive UTRs showed that tri- and di-nucleotides occurred in $35 \%$ and $24 \%$, respectively. In CDSs, trinucleotide motifs were predominant $(33 \%)$. This result is in accordance with previous studies, which showed that selection against frameshift mutations limits nontriplet SSRs expansion in CDS regions (Metzgar et al., 2002).

In this study, we have demonstrated that a novel set of 46 EST-SSRs has good diversity properties, and we are convinced that it will be helpful in olive breeding programs, in the construction of linkage maps and it will aid in elucidating some biochemical pathways and physiological processes in olives. Furthermore, due to their possible transferability to the related species, ESTSSRs can also be used in the field of comparative genomics. All sequencing data and developed primer pairs for genic markers are available to the olive community and all interested research groups through public NCBI databases.

\section{References}

Adawy, S.S.; Mokhtar, M.M.; Alsamman, A.M.; Sakr, M.M. 2015. Development of annotated EST-SSR database in olive (Olea europaea). Internation Journal of Science and Research 4: 10631073.

Alagna, F.; D'Agostino, N.; Torchia, L.; Servili, M.; Rao, R.; Pietrella, M.; Giuliano, G.; Chiusana, M.L.; Baldoni, L.; Perrotta, G. 2009. Comparative 454 pyrosequencing of transcripts from two olive genotypes during fruit development. BMC Genomics 10: 399.

Ali Hashmi, M.; Khan, A.; Hanif, M.; Fatooq, U.; Perveen, S. 2015. Traditional uses, phytochemistry, and pharmacology of Olea europaea (Olive). Evidence-Based Complementary and Alternative Medicine 2015: ID 541591.

Bandelj, D.; Jakše, J.; Javornik, B. 2004. Assessment of genetic variability of olive varieties by microsatellite and AFLP markers. Euphytica 136: 93-102.

Caroli, S.; Santoni, S.; Ronfort, J. 2011. AMaCAID: a useful tool for accurate marker choice for accession identification and discrimination. Molecular Ecology Resources 11: 733-738.

Chen, C.; Zhou, P.; Choi, Y.A.; Huang, S.; Gmitter, F.G.J. 2006. Mining and characterizing microsatellites from citrus ESTs. Theoretical and Applied Genetics 112: 1248-1257.

Chen, C.; Bock, C.H.; Beckman, T.G. 2014. Sequence analysis reveals genomic factors affecting EST-SSR primer performance and polymorphism. Molecular Genetics and Genomics 289: 1147-1156.

Cicerale, S.; Conlan, X.A.; Sinclair, A.J.; Keast, R.S. 2009. Chemistry and health of olive oil phenolics. Critical Reviews in Food Science and Nutrition 49: 218-236. 
Cipriani, G.; Spadotto, A.; Jurman, I.; Di Gaspero, G.; Crespan, M.; Meneghetti, S.; Frare, E.; Vignani, R.; Cresti, M.; Morgante, M.; Pezzotti, M.; Pe, E.; Policriti, A.; Testolin, R. 2010. The SSR-based molecular profile of 1005 grapevine (Vitis vinifera L.) accessions uncovers new synonymy and parentages, and reveals a large admixture amongst varieties of different geographic origin. Theoretical and Applied Genetics 121: 15691585.

Corrado, G. 2016. Advances in DNA typing in the agro-food supply chain. Trends in Food Science \& Technology 52: 80-89.

Dakin, E.E.; Avise, J.C. 2004. Microsatellite null alleles in parentage analysis. Heredity 93: 504-509.

De la Rosa, R.; Belaj, A.; Muñoz-Mérida, A.; Trelles, O.; OrtízMartín, I.; González-Plaza, J.J.; Valpuesta, V.; Beuzón, C.R. 2013. Development of EST-derived SSR markers with long-core repeat in olive and their use for paternity testing. Journal of the American Society for Horticultural Science 138: 290-296.

Dillon, N.L.; Innes, D.J.; Bally, I.S.E.; Wright, C.L.; Devitt, L.C.; Dietzgen, A.G. 2014. Expressed sequence tag-simple sequence repeat (EST-SSR) marker resources for diversity analysis of mango (Mangifera indica L.). Diversity 6: 72-87.

Essalouh, L.; El Aabidine, Z.; Contreras, S.; Ben Sadok, I.; Santoni, S.; Khadari, B.; Jakse, J.; Bandelj, D. 2014. Genomic and EST microsatellite loci development and use in olive: molecular tools for genetic mapping and association studies. Acta Horticulturae 1057: 543-550.

Fujimori, S.; Washio, T.; Higo, K.; Ohtomo, Y.; Murakami, K.; Matsubara, K.; Kawai, J.; Carninci, P.; Hayashizaki, Y.; Kikuchi, S.; Tomita, M. 2003. A novel feature of microsatellites in plants: a distribution gradient along the direction of transcription. FEBS Letters 554: 17-22.

Ghanbari, R.; Anwar, F.; Alkharfy, K.M.; Gilani, A.H.; Saari, N. 2012. Valuable nutrients and functional bioactives in different parts of olive (Olea europaea L.): a review. International Journal of Molecular Sciences 13: 3291-3340.

Gupta, P.K.; Rustgi, S.; Sharma, S.; Singh, R.; Kumar, N.; Balyan, S. 2003. Transferable EST-SSR markers for the study of polymorphism and genetic diversity in bread wheat. Molecular Genetics and Genomics 270: 315-323.

Huang, H.; Lu, J.; Ren, Z.; Hunter, W.; Dowd, S.E.; Dang, P. 2011. Mining and validating grape (Vitis L.) ESTs to develop EST-SSR markers for genotyping and mapping. Molecular Breeding 28: 241-254.

Jian, Z.H.; Liu, X.S.; Hu, J.B.; Chen, Y.H.; Feng, J.C. 2012. Mining microsatellite markers from public expressed sequence tag sequences for genetic diversity analysis in pomegranate. Journal of Genetics 9: 353-358.

Jiang, G.L. 2013. Molecular markers and marker-assisted breeding in plants. p. 45-84. In: Andersen, S.N., ed. Plant breeding from laboratories to fields. InTech, Rijeka, Croatia. DOI: http:// dx.doi.org/10.5772/52583.

Jung, S.; Abbott, A.; Jesudurai, C.; Tomkins, J.; Main, D. 2005. Frequency, type, distribution and annotation of simple sequence repeats in Rosaceae ESTs. Functional and Integrative Genomics 5: 136-143.

Kalia, R.K.; Rai, M.K.; Kali, S.; Singh, R.; Dhawan, A.K. 2011. Microsatellite markers: an overview of the recent progress in plants. Euphytica 177: 309-334.
Kanehisa, M.; Goto, S. 2000. KEGG: Kyoto Encyclopedia of Genes and Genomes. Nucleic Acids Research 28: 27-30.

Koressaar, T.; Remm, M. 2007. Enhancements and modifications of primer design program Primer3. Bioinformatics 23: 12891291.

Kump, B.; Javornik, B. 1996. Evaluation of genetic variability among common buckwheat (Fagopyrum esculentum Moench.) populations by RAPD markers. Plant Science 114: 149-158.

La Malfa, S.; Curró, S.; Douglas, A.B.; Brugaletta, M.; Caruso, M.; Gentile, A. 2014. Genetic diversity revealed by EST-SSR markers in carob tree (Ceratonia siliqua L.). Biochemical Systematics and Ecology 55: 205-211.

Lavee, S.; Avidan, B.; Ben-Ari, G. 2014. Trends in breeding new olive varieties in Israel for quality and economic management. Agricultural Science 5: 701-709.

Levene, H. 1949. On a matching problem in genetics. The Annals of Mathematical Statistics 20: 91-94.

Li, Y.C.; Korol, A.B.; Fahima, T.; Nevo, E. 2004. Microsatellite within genes: structure, function and evolution. Molecular Biology and Evolution 21: 991-1007.

Liu, S.R.; Li, W.Y.; Long, D.; Hu, C.G.; Zhang, J.Z. 2013. Development and characterization of genomic and expressed SSRs in citrus by genome-wide analysis. PloS One 8: e75149.

Mantel, N. 1967. The detection of diseases clustering and a generalized regression approach. Cancer Research 27: 209-220.

Metzgar, D.; Liu, L.; Hansen, C.; Dybvig, K.; Wills, C. 2002. Domain level differences in microsatellite distribution and content result from different relative rates of insertion and deletion mutations. Genome Research 12: 408-413.

Mignone, F.; Gissi, C.; Liuni, S.; Pesole, G. 2002. Untranslated regions of mRNAs. Genome Biology 3: reviews0004.1-0004.10.

Milne, I.; Bayer, M.; Cardle, L.; Shaw, P.; Stephen, G.; Wright, F.; Marshall, D. 2010. Tablet-next generation sequence assembly visualization. Bioinformatics 26: 401-402.

Muñoz-Mérida, A.; González-Plaza, J.J.; Cañada, A.; Blanco, A.M.; García-López, M.D.; Rodríguez, J.M.; Pedrola, L.; Sicardo, M.D.; Hernández, M.L.; De la Rosa, R.; Belaj, A.; Gil-Borja, M.; Luque, F.; Martínez-Rivas, J.M.; Pisano, D.G.; Trelles, O.; Valpuesta, V.; Beuzón, C.R. 2013. De Novo assembly and functional annotation of the olive (Olea europaea) transcriptome. DNA Research 20: 93-108.

Ozgenturk, N.O.; Oruc, F.; Sezerman, U.; Kucukural, A.; Korkut, S.V.; Toksoz, F.; Cemal, U. 2010. Generation and analysis of expressed sequence tags from Olea europaea L. Comparative and Functional Genomics 2010: ID 757512.

Pasqualone, A.; Montemurro, C.; di Rienzo, V.; Summo, C.; Paradiso, V.M.; Caponio, F. 2016. Evolution and perspectives of cultivar identification and traceability from tree to oil and table olives by means of DNA markers. Journal of the Science of Food and Agriculture 96: 3642-3657.

Rešetič, T.; Štajner, N.; Bandelj, D.; Javornik, B.; Jakše, J. 2013. Validation of candidate reference genes in RT-qPCR studies of developing olive fruit and expression analysis of four genes involved in fatty acids metabolism. Molecular Breeding 32: 211-222.

Rudd, S. 2003. Expressed sequence tags: alternative or complement to whole genome sequences? Trends in Plant Science 8: 321-329. 
Schuelke, M. 2000. An economic method for the fluorescent labelling of PCR fragments. Nature Biotechnology 18: 233-234.

Toth, G.; Gaspari, Z.; Jurka, J. 2000. Microsatellite in different eukaryotic genomes: survey and analysis. Genome Research 10: 967-981.

Untergrasser, A.; Cutcutache, I.; Koressaar, T.; Ye, J.; Faircloth, B.C.; Remm, M.; Rozen, S.G. 2012. Primer3: new capabilities and interfaces. Nucleic Acids Research 40: e115.

Varshney, R.K.; Graner, A.; Sorrells, M.E. 2005. Genic microsatellite markers in plants: features and applications. Trends in Biotechnology 23: 48-55.

Vendramin, E.; Dettori, M.T.; Giovinazzi, J.; Micali, S.; Quarta, R.; Verde, I. 2007. A set of EST-SSRs isolated from peach fruit transcriptome and their transportability across Prunus species. Molecular Ecology Resources 7: 307-310.
Zhang, H.; Wei, L.; Miao, H.; Zhang, T.; Wang, C. 2012. Development and validation of genic-SSR markers in sesame by RNA-seq. BMC Genomics 13: 316.

Zhang, M.; Fan, L.; Liu, O.; Song, Y.; Wei, S.; Zhang, S.; Wu, J. 2013. A novel set of EST-derived SSR markers for pear and cross-species transferability in Rosaceae. Plant Molecular Biology Reporter 32: 290-302.

Zheng, Y.; Zhao, L.J.; Gao, J.P.; Fei, Z.J. 2011. iAssembler: a package for de novo assembly of Roche-454/Sanger transcriptome sequences. BMC Bioinformatics 12: 453. 